\title{
Frequency of Sustained Intracranial Pressure Elevation during Treatment of Severe Intraventricular Hemorrhage
}

\author{
Wendy C. Ziai ${ }^{a} \quad$ Michel T. Torbey ${ }^{a} \quad$ Neal J. Naff ${ }^{b}$ Michael A. Williams ${ }^{a}$ \\ Ross Bullock ${ }^{c}$ Anthony Marmarou ${ }^{c}$ Stanley Tuhrim ${ }^{d}$ Eric Schmutzhard ${ }^{\mathrm{e}}$ \\ Bettina Pfausler ${ }^{\mathrm{e}}$ Daniel F. Hanley ${ }^{\mathrm{a}}$ \\ Departments of a Neurology and ${ }^{b}$ Neurosurgery, Johns Hopkins University School of Medicine, Baltimore, Md., \\ 'Department of Neurological Surgery, Medical College of Virginia, Richmond, Va., and dDepartment of Neurology, \\ Mount Sinai Medical Center, New York, N.Y., USA; e Department of Neurology, University Hospital, Innsbruck, Austria
}

\section{Key Words}

Intracerebral hemorrhage $\cdot$ Thrombolysis - Intracranial pressure $\cdot$ Stroke $\cdot$ Hydrocephalus

\begin{abstract}
Background: Elevated intracranial pressure (ICP) is an important marker of neurological deterioration. The occurrence and significance of elevated ICP and low cerebral perfusion pressure (CPP) in aggressively treated spontaneous intraventricular hemorrhage (IVH) are not defined. Methods: We performed a secondary longitudinal exploratory data analysis of a randomized multicenter trial of urokinase (UK) versus placebo (Pcb) as a treatment for IVH. Eleven IVH patients who required an external ventricular drain (EVD) were randomized to receive either intraventricular UK or Pcb every $12 \mathrm{~h}$ until clinical response permitted EVD removal. ICP and CPP were recorded every 4 or $6 \mathrm{~h}$, as well as before and $1 \mathrm{~h}$ after EVD closure for administration of study agent. ICP, CPP and the proportion of ICP readings above 20, 30, 40 and $50 \mathrm{~mm} \mathrm{Hg}$ were analyzed. Results: Six UK and 5 Pcb patients aged 39-74 years (mean \pm standard deviation; $53 \pm 11$ years) were enrolled. Initial ICP ranged from 0 to $38 \mathrm{~mm} \mathrm{Hg}$ (10.9 \pm 11.0), initial CPP from 65 to $133 \mathrm{~mm} \mathrm{Hg}$ (100.5 \pm 17.7).
\end{abstract}

We recorded 472 ICP readings over the entire monitoring period. Of these 65 (14\%) were > $20 \mathrm{~mm} \mathrm{Hg}, 23$ (5\%)>30 $\mathrm{mm} \mathrm{Hg}$, $9(2 \%)>40 \mathrm{~mm} \mathrm{Hg}$ and $3(<1 \%)>50 \mathrm{~mm} \mathrm{Hg}$. Only 2 of 141 intraventricular injections of study agent with EVD closure were not tolerated and required reopening of the EVD. Conclusions: In the intensive care unit, initial ICP measured with an EVD was uncommonly elevated (1/11 patients) in this group of severe IVH patients despite acute obstructive hydrocephalus. Frequent monitoring reveals ICP elevation $>20$ $\mathrm{mm} \mathrm{Hg}$ in $14 \%$ of observations during use of EVD. ICP elevation, though it can occur, is not routinely associated with EVD closure for thrombolytic treatment with UK.

Copyright $\odot 2009$ S. Karger AG, Basel

\section{Introduction}

Intraventricular hemorrhage (IVH) carries a high morbidity and mortality, with over $80 \%$ mortality in cases involving hemorrhage in all four ventricles [1-3]. In both the international Surgical Trial in Intracerebral Hemorrhage (STICH) study and the multicenter, randomized, placebo (Pcb)-controlled trial on the effectiveness of rFVIIa in spontaneous intracerebral hemorrhage

\section{KARGER}

(c) 2009 S. Karger AG, Basel

Fax +41613061234 E-Mail karger@karger.ch www.karger.com www.karger.com/ced
Wendy C. Ziai, MD

Johns Hopkins Hospital, Division of Neurosciences Critical Care 600 N. Wolfe Street/Meyer 8-140

Baltimore, MD 21287 (USA)

Tel. +1 410614 6121, Fax +1 410614 7903, E-Mail weziai@jhmi.edu 
(ICH), IVH was identified as a significant outcome severity factor $[4,5]$.

Intracranial hypertension is thought to contribute to an altered level of consciousness in patients with IVH by several mechanisms: an acute reduction in cerebral perfusion pressure (CPP), ischemic encephalopathy [6], diffuse cerebral edema, and compression of the rostral brainstem and thalamus by an expanded $3 r$ d ventricle [7]. Volume of intraventricular blood and degree of obstructive hydrocephalus are reported as prognostic factors in IVH $[8,9]$. Although external ventricular drainage (EVD) is considered life-saving in patients with neurological deterioration secondary to acute hydrocephalus, no relationship between intracranial pressure (ICP) elevation or ICP control and neurological deterioration or improvement has been established in IVH [10]. The occurrence of elevated ICP in IVH may be less common than expected from other causes of acute hydrocephalus [11].

The objective of this exploratory analysis was to analyze the frequency, extent, and significance of intracranial hypertension in a prospectively defined, severe IVH patient population managed with high intensity intensive care unit (ICU) care during the initial week of illness. Specific questions of interest were: does initial ICP elevated in patients with IVH require an EVD for hydrocephalus?; does ICP differ over time in patients treated with intraventricular urokinase (UK) and Pcb?, and does clamping the EVD for $1 \mathrm{~h}$ after test article injection cause potentially harmful ICP elevation?

\section{Materials and Methods}

The primary results of this Pcb-controlled double-blind study of intraventricular UK for IVH have been previously reported [12]. Eight patients from the Johns Hopkins Hospital and 3 patients from University Hospital, Innsbruck, Austria with acute IVH (within $48 \mathrm{~h}$ of onset) requiring an EVD for treatment of obstructive hydrocephalus were randomized to receive intraventricularly either 1-ml Pcb injections of normal saline (sterile nonbacteriostatic $0.9 \% \mathrm{NaCl}$ ) every $12 \mathrm{~h}$ (Pcb group: 5 patients) or injections of UK (Abbodinase; Abbott Laboratories; 25,000 IU) in $1 \mathrm{ml}$ of normal saline solution every $12 \mathrm{~h}$ (UK group: 6 patients). Informed consent was sought only after EVD had been inserted. Exclusion criteria included: posterior fossa hematoma; intraparenchymal hemorrhage volume $>30 \mathrm{ml}$ (by ABC/2 method); suspected intracerebral aneurysm or arteriovenous malformation (excluded by appropriate diagnostic studies); any severe complicating illness; active internal bleeding, current use of heparin, coagulopathy with PT or PTT outside of normal range, or thrombocytopenia with platelet count $<75 \mathrm{IU} / \mathrm{mm}^{3}$; pregnancy, and age $<18$ years. Patients were admitted to an ICU with staff experienced in the acute care of patients with IVH and EVDs. Other treatments for ICP management such as hyperventilation, mannitol, and hypertonic saline were administered by the treating physician for sustained ICP greater than $20 \mathrm{~mm} \mathrm{Hg}$.

All patients with a ventricular catheter (Codman external drainage system II, Codman \& Shurtleff, Randolf, Mass., USA) inserted in the initial $24 \mathrm{~h}$ of illness to treat IVH were approached for randomization during 1995-1999. EVDs were placed into the frontal horn of the lateral ventricle and tunneled under the scalp by neurosurgical staff. Intraventricular location of the catheter tip was confirmed by ICP waveform morphology and by CT scan. The transducer was placed at the level of the foramina of Monro (external landmark: external auditory meatus). Patients were treated with intravenous oxacillin (1 g intravenously, q6h) prophylactically. Mean arterial pressure was measured continuously by a radial arterial catheter. ICP and CPP were monitored continuously. ICP and CPP were recorded at baseline and at 4- or 6hour intervals depending on the study site. For each ICP measurement, the EVD was closed for $10 \mathrm{~min}$ and the ICP was recorded at the end of this time period. High ICP events were defined at four thresholds as ICP above 20,30, 40 or $50 \mathrm{~mm} \mathrm{Hg}$ for at least 5 min.

ICP and CPP were also recorded before, during, and after injection of the study agent. After each injection, the EVD was closed for $1 \mathrm{~h}$ to allow adequate time for study agent-clot interaction. The EVD was reopened within the initial hour only if needed to control medically intractable ICP elevation (ICP >20 mm $\mathrm{Hg}$ refractory to hyperventilation and mannitol administration). After $1 \mathrm{~h}$, the EVD was reopened with a drainage gradient specified by the treating physician $(0-15 \mathrm{~mm} \mathrm{Hg})$. ICP elevations during drug administration were designated as a serious event only if they were treated unsuccessfully and remained elevated after reopening the EVD. This occurred in only 2 instances. Flushing of the intraventricular portion of the catheter was performed with each injection and also in response to loss of the waveform secondary to thrombus occluding the catheter. Replacement of the catheter for obstruction was not documented in any patient. Failure to control ICP resulted in a CT scan to evaluate for structural causes such as trapped ventricles, inadequate catheter position and edema.

The routine for monitoring, CSF drainage, and removal of EVDs was standardized in both ICUs based on a prospectively determined protocol. The daily CSF volume drained was recorded. Catheters were not flushed except as described above. The study agent was administered every $12 \mathrm{~h}$ until the EVD was removed, based on patient tolerance of EVD closure for $24 \mathrm{~h}$ (i.e. no sustained ICP elevation $>20 \mathrm{~mm} \mathrm{Hg}$ ).

$\mathrm{CPP}$ was recorded for each ICP measurement. Low CPP events were defined at two thresholds, $\mathrm{CPP}<60$ or $<70 \mathrm{~mm} \mathrm{Hg}$. CPP $<60$ $\mathrm{mm} \mathrm{Hg}$ and ICP $>20 \mathrm{~mm} \mathrm{Hg}$ were considered threshold levels for treatment based on Brain Trauma Foundation guidelines [13, 14].

Head CT scans were obtained per protocol on alternate days, and additional CT studies were performed in the event of acute neurological deterioration. The volumes of intraventricular and intraparenchymal hematomas were measured independently by a neuroradiologist using standard computerized volumetric analysis as described by Steiner et al. [15]. Clearance of blood from the 3rd and 4th ventricles (ability to visualize a clear pathway of hypodense CSF through the lower ventricular system) was assessed independently and blindly by MT and WZ. This protocol was approved by the Internal Review Boards of both institutions. 
Table 1. Baseline characteristics

\begin{tabular}{lccc}
\hline Factor & $\begin{array}{l}\text { UK group } \\
(\mathrm{n}=6)\end{array}$ & $\begin{array}{l}\text { Placebo group } \\
(\mathrm{n}=5)\end{array}$ & $\begin{array}{l}\mathrm{p} \\
\text { value }\end{array}$ \\
\hline Age (range) & $52 \pm 6(39-74)$ & $55 \pm 2(50-61)$ & $\mathrm{NS}$ \\
Gender (M/F) & $4 / 2$ & $3 / 2$ & $\mathrm{NS}$ \\
Race (C/AA/H) & $4 / 1 / 1$ & $2 / 2 / 1$ & $\mathrm{NS}$ \\
Hypertension history & 4 & 4 & $\mathrm{NS}$ \\
Diabetes mellitus history & 2 & 0 & $\mathrm{NS}$ \\
Initial IVH volume, ml & $67 \pm 24$ & $42 \pm 25$ & $\mathrm{NS}$ \\
Initial ICH volume, ml & $4 \pm 4$ & $13 \pm 7$ & 0.02 \\
\hline
\end{tabular}

Age and volume are given as mean \pm SD. $t$ test or Fisher's exact test. NS $=$ Nonsignificant; $\mathrm{C}=$ Caucasian; $\mathrm{AA}=$ African-American; $\mathrm{H}=$ Hispanic.

\section{Statistical Analysis}

Demographic variables, baseline IVH and ICH volumes, total duration of ICP monitoring, and differences in mean ICP before and after injection of study agent and before and after blood clearance from the $3 \mathrm{rd}$ and 4 th ventricles were compared between UKand Pcb-treated patients. Wilcoxon rank-sum test was used to compare variables with nonnormal distributions. Student's t test was used for continuous variables with normal distributions and $\chi^{2}$ or Fisher's exact test was used for analysis of categorical data as appropriate. Correlations were performed using the Spearman correlation test. All ICP elevation events $>20,30,40$ and $50 \mathrm{~mm}$ $\mathrm{Hg}$ were tabulated such that $>20 \mathrm{~mm} \mathrm{Hg}$ events included all ICPs above these thresholds. For longitudinal data analysis, including ICP and CPP levels, the number of ICP events above critical ICP thresholds $(>20,30,40$ and $50 \mathrm{~mm} \mathrm{Hg}$ ), and daily CSF volume drained, we compared UK- and Pcb-treated patients for the first 5 days of monitoring using multiple regression techniques for longitudinal data. We chose to analyze the first 5 days of ICP/CPP monitoring as all patients (except 1 who died) had EVDs in place during this time period and we would have a similar number of observations per patient. Linear and logistic regression models were developed regressing the outcomes of ICP, CPP, and the binary outcome of critical ICP values by treatment group and for the entire group against time that appropriately took into account within-person correlation of ICP/CPP measurements (the method of general estimating equations). We also developed models regressing daily CSF volumes drained by treatment group against time and initial IVH volume. Statistical significance was assigned for $p<0.05$. Data are presented as mean $\pm S D$, unless otherwise indicated. No correction for multiple tests was performed as all analysis was deemed exploratory.

\section{Results}

\section{Patient Characteristics}

Eleven patients with an age range of 39-74 years (mean $53 \pm 11$ years) were enrolled. Randomization groups did not differ with respect to age, gender, race or stroke risk factors (table 1).

Intracranial Pressure and Intraventricular Hemorrhage

\section{EVD Placement}

All patients had EVDs inserted within $12 \mathrm{~h}$ of onset of IVH, except 2 patients whose EVDs were placed at 24$36 \mathrm{~h}$ after onset. The EVD was inserted in the right frontal horn in 7 patients, and in the left in 4 . The EVD was placed in the ventricle with the least blood in all but 2 patients.

\section{Initial ICP, ICH and IVH Volume}

Initial mean ICP at the time of EVD insertion was 11 $\pm 11 \mathrm{~mm} \mathrm{Hg}$ (range $0-38 \mathrm{~mm} \mathrm{Hg}$ ). Only 1 patient had initial ICP $>20 \mathrm{~mm} \mathrm{Hg}$ (table 2; fig. 1). Due to CSF loss at the time of EVD insertion, especially if ICP is elevated, the recorded ICP may not reflect actual ICP before insertion of the EVD. The initial mean ICH volume was greater in the Pcb group although this difference may not be clinically significant. Three patients in the UK group had no measurable intraparenchymal component of blood. IVH volume was not different between groups. There was no correlation between initial IVH or ICH volume and initial ICP (Spearman's rho $=-0.02 ; \mathrm{p}=0.96 ; \mathrm{rho}=-0.19$; $\mathrm{p}=0.57$, respectively). Neither initial IVH volume nor ICH volume were correlated with time to clearance of blood from the 3rd and 4th ventricles (Spearman's rho = $-0.44 ; \mathrm{p}=0.18 ;$ rho $=0.22 ; \mathrm{p}=0.52$, respectively). During the first 5 days of ICP monitoring linear regression analysis showed no significant relationship between mean daily ICP levels and initial IVH volume or initial ICH volume ( $\mathrm{p}=0.91$ and $\mathrm{p}=0.62$, respectively).

\section{ICP and Duration of ICP Monitoring}

The mean duration of ICP monitoring was $164 \pm$ $123 \mathrm{~h}$ (range 44-460 h), and was not statistically different between groups (table 2). Only 4 patients required ICP monitoring for more than 7 days; the EVD of the last UK patient was removed on day 7 , that of the last Pcb patient on day 19. 
Fig. 1. Scatterplot comparing distribution of ICP readings for Pcb- and UK-treated patients across the first 5 days of monitoring. Horizontal lines indicate median ICP for each group $(\mathrm{UK}=11 \mathrm{~mm} \mathrm{Hg}$; $\mathrm{Pcb}=12$ $\mathrm{mm} \mathrm{Hg}$ ). The median daily ICP never exceeded $20 \mathrm{~mm} \mathrm{Hg}$ although individual readings $>20 \mathrm{~mm} \mathrm{Hg}$ are observed more frequently in the Pcb-treated group.

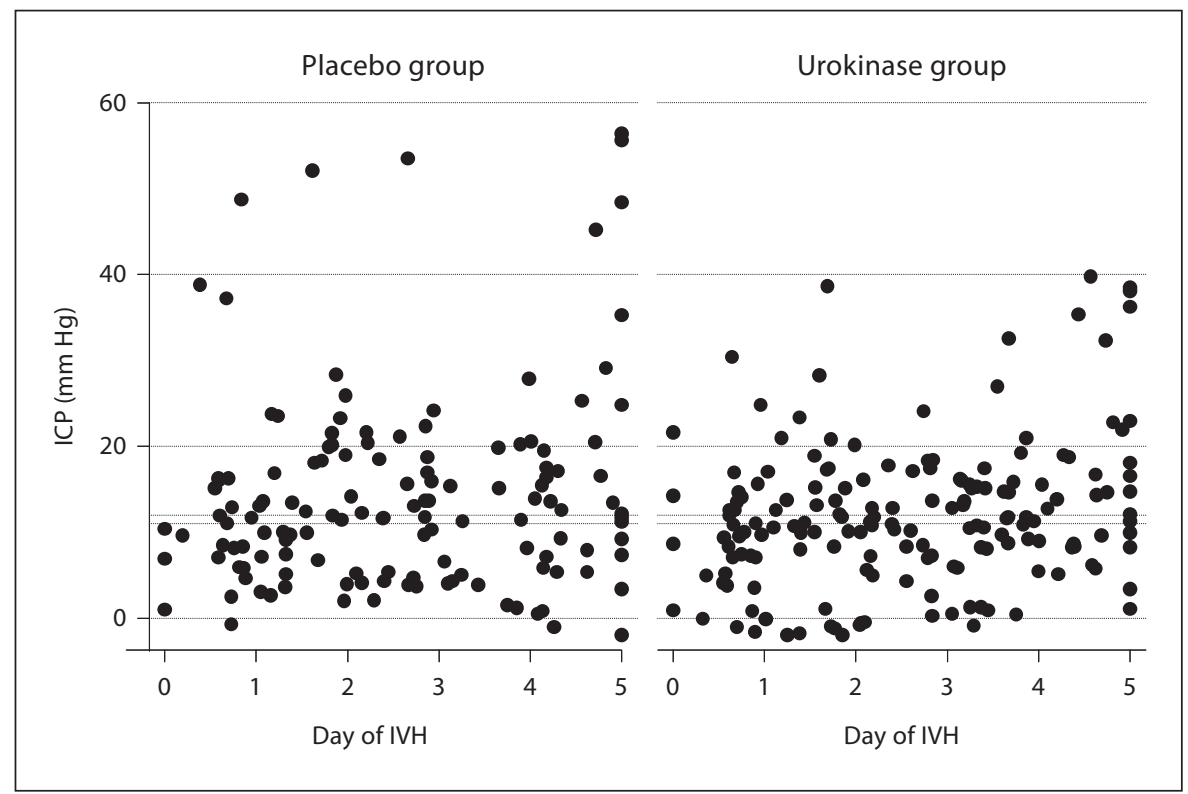

Table 2. Comparison of UK and placebo group during treatment

\begin{tabular}{|c|c|c|c|}
\hline Factor & $\begin{array}{l}\text { UK group } \\
(\mathrm{n}=6)\end{array}$ & $\begin{array}{l}\text { Placebo group } \\
(n=5)\end{array}$ & $\mathrm{p}$ value \\
\hline Initial $\mathrm{ICP}^{1}, \mathrm{~mm} \mathrm{Hg}$ & $8.3 \pm 8.5$ & $14.0 \pm 13.8$ & NS \\
\hline Mean duration of ICP monitoring ${ }^{2}, \mathrm{~h}$ & $116 \pm 30$ & $221 \pm 172$ & NS \\
\hline Mean time of blood clearance from $3 \mathrm{rd} / 4$ th ventricles ${ }^{2}$, days & $3.8 \pm 1.6$ & $10.6 \pm 0.6$ & 0.02 \\
\hline Mean ICP before clearance of $3 \mathrm{rd} / 4$ th ventricles ${ }^{2}, \mathrm{~mm} \mathrm{Hg}$ & $10.5 \pm 7.7$ & $15.6 \pm 10.7$ & NS \\
\hline Mean ICP after clearance of $3 \mathrm{rd} / 4$ th ventricles $^{2}, \mathrm{~mm} \mathrm{Hg}^{\circ}$ & $13.0 \pm 8.8$ & $11.9 \pm 7.1$ & $<0.001$ \\
\hline Mean change in ICP from preinjection to $1 \mathrm{~h}$ postinjection & & & $<0.01$ \\
\hline of study agent ${ }^{2}[\mathrm{ICP}$ (postinj.) - ICP(preinj.)], mm Hg & $3.1 \pm 1.0$ & $8.2 \pm 1.3$ & \\
\hline Patients with blood cleared from $3 \mathrm{rd} / 4$ th ventricles ${ }^{1}, \mathrm{n}$ & $6 / 6$ & $2 / 5$ & NS \\
\hline
\end{tabular}

Values represent mean $\pm \mathrm{SD}$ or number. $\mathrm{SD}=$ Standard deviation; $\mathrm{NS}=$ nonsignificant.

${ }^{1} \mathrm{t}$ test or Fisher's exact test.

${ }^{2}$ Wilcoxon rank-sum test.

Individual patient mean daily ICP rarely exceeded 25 $\mathrm{mm}$ Hg. Three patients (1 UK- and 2 Pcb-treated) had no ICP readings $>20 \mathrm{~mm} \mathrm{Hg}$ for the duration of their EVD. During the entire monitoring period, there were $65 \mathrm{ICP}$ recordings over $20 \mathrm{~mm} \mathrm{Hg}$ (14\% of ICP recordings), 23 events over $30 \mathrm{~mm} \mathrm{Hg}(5 \%), 9$ over $40 \mathrm{~mm} \mathrm{Hg}(2 \%)$, and only 3 over $50 \mathrm{~mm} \mathrm{Hg}$ (0.6\%) (fig. 1). During the first 5 days of monitoring, the number of ICP readings over 20 $\mathrm{mm} \mathrm{Hg}$ was not different between groups $\left(\chi^{2}=3.06\right.$; $\mathrm{p}=$ 0.08 ). During the first 5 days linear regression analysis showed a significant increase in ICP levels over time in the UK-treated group ( $\mathrm{p}<0.001)$, but not in the Pcb group $(\mathrm{p}=0.49)$. Analysis of the model with both groups showed a small, but significant increase in ICP over the first 5 days $(\mathrm{p}=0.002)$, but no significant difference by treatment group $(\mathrm{p}=0.48)$. Analysis of above-threshold ICP events (for ICP greater than 20,30, 40 and $50 \mathrm{~mm} \mathrm{Hg}$ ) showed statistically significant increases in the probability of threshold events with time for only the UK group which occurred only for the lower thresholds of 20 and $30 \mathrm{~mm} \mathrm{Hg}$ (table 3). Analysis with both groups in the model showed no significant differences by time or group for threshold events $>20,30,40$ or $50 \mathrm{~mm} \mathrm{Hg}$. When stratified by day of ICP monitoring, the proportion of 
Fig. 2. Box plot comparing daily CSF drainage in Pcb- and UK-treated group for the first 5 days of monitoring. CSF drainage was greater for the Pcb group on all days, but was not significantly different. Times of removal of EVDs in the UK-treated (mean 6 days) and Pcb-treated (mean 10 days) group were not significantly different $(\mathrm{p}=0.21)$. Box plots include multiple observations per patient. The single data point shown for the day $3 \mathrm{UK}$ group is an outlier. Note: sample size decreases over time due to removal of EVDs.

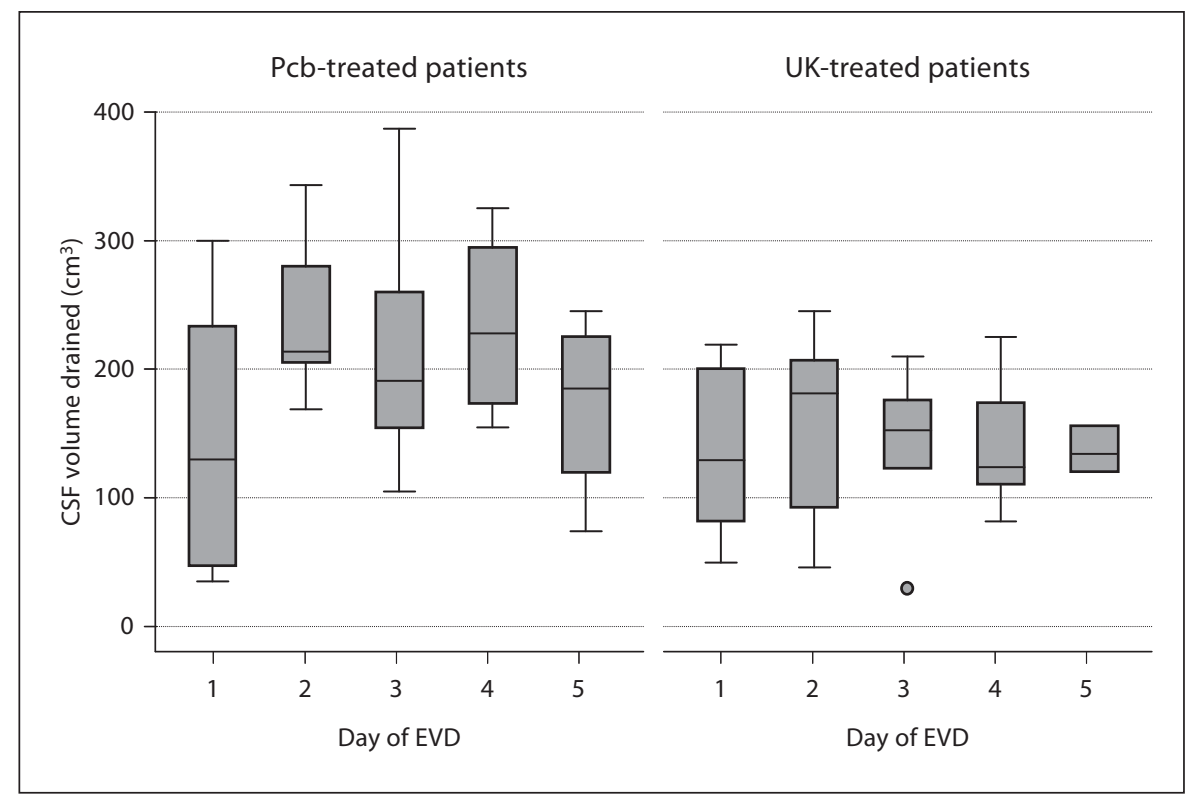

Table 3. Results of logistic regression models regressing critical ICP levels by treatment group over time for the first 5 days of ICP monitoring

\begin{tabular}{llclc}
\hline $\begin{array}{l}\text { ICP } \\
\text { threshold } \\
\mathrm{mm} \mathrm{Hg}\end{array}$ & UK group $(\mathrm{n}=6)$ & $\mathrm{p}$ value & $\begin{array}{l}\text { Placebo group } \\
(\mathrm{n}=5)\end{array}$ & $\mathrm{p}$ value \\
\hline$>20$ & $1.02(1.00,1.04)$ & 0.01 & $1.00(0.99,1.02)$ & 0.44 \\
$>30$ & $1.04(1.01,1.07)$ & 0.01 & $1.00(0.99,1.02)$ & 0.58 \\
$>40$ & $1.06(0.96,1.16)$ & 0.26 & $1.02(0.99,1.04)$ & 0.22 \\
$>50$ & no prediction available & $1.04(0.99,1.08)$ & 0.10 \\
\hline
\end{tabular}

Values represent odds ratio $(95 \% \mathrm{CI})$.

high ICP events (>20 mm Hg) was greatest at day 5 for both groups. These results are consistent with a 'weaning effect' moving the ICP trend slightly upwards.

\section{Tolerance to EVD Closure}

Of a total of 141 intraventricular injections of study agent, the 1-hour EVD closure time was tolerated without reopening in all but 2 instances. There were, however, 28 injections associated with transient elevation of ICP $>20$ $\mathrm{mm} \mathrm{Hg}$ during the 1-hour IVC closure time, 24 of which occurred in a single patient in the Pcb group. Ninetythree percent $(26 / 28)$ of ICP elevations during EVD closure for drug administration were minor and did not require opening. The mean change (increase) in ICP from immediately before closing the EVD for injection of study
Table 4. Comparison of mean ICP of individual patients before and after radiographic clearance of blood from the $3 \mathrm{rd}$ and 4 th ventricles

\begin{tabular}{lcc}
\hline $\begin{array}{l}\text { Patient/ } \\
\text { study group }\end{array}$ & $\begin{array}{l}\text { Mean ICP } \\
\text { before clearance } \\
\text { mm Hg }\end{array}$ & $\begin{array}{l}\text { Mean ICP } \\
\text { after clearance } \\
\text { mm Hg }\end{array}$ \\
\hline //UK & $9.8 \pm 6.2$ & $13.1 \pm 5.1$ \\
$2 / \mathrm{UK}$ & $10.9 \pm 6.4$ & $15.2 \pm 12.9$ \\
$3 / \mathrm{UK}$ & $12.8 \pm 8.1$ & $8.8 \pm 7.6$ \\
//UK & $7.4 \pm 9.1$ & $1.6 \pm 2.5$ \\
$5 / \mathrm{UK}$ & $8.5 \pm 4.4$ & $13.7 \pm 10.8$ \\
$6 / \mathrm{UK}$ & $14.3 \pm 3.6$ & $15.2 \pm 3.2$ \\
1/Pcb & $8.9 \pm 8.2$ & - \\
2/Pcb & $14.0 \pm 6.1$ & - \\
$3 / \mathrm{Pcb}$ & $14.4 \pm 8.7$ & $15.2 \pm 6.6$ \\
$4 / \mathrm{Pcb}$ & $5.2 \pm 4.0$ & - \\
$5 / \mathrm{Pcb}$ & $23.6 \pm 11.9$ & $14.7 \pm 7.4$ \\
\hline
\end{tabular}

- = No ICP recordings as 3rd and 4th ventricles did not clear by the end of the monitoring period.

agent, until just before reopening after the 1-hour closing time was $3.1 \pm 1.0 \mathrm{~mm} \mathrm{Hg}$ in the UK group and $8.2 \pm$ $1.3 \mathrm{~mm} \mathrm{Hg}$ in the Pcb group. This was significantly lower in the UK group $(\mathrm{p}<0.01)$.

\section{Cerebrospinal Fluid Volume Drainage}

During the first 5 days of EVD use linear regression analysis showed a significant decrease in daily CSF vol- 
ume drained over time in the UK-treated group ( $\mathrm{p}=$ $0.02)$, but not in the Pcb group ( $\mathrm{p}=0.70)$ (fig. 2$)$. Analysis of the model for all subjects showed that daily CSF volume drained was not significantly associated with baseline IVH volume and did not differ statistically by treatment group ( $p=0.24$ and 0.36 , respectively). We did not adjust for daily IVH volume because not all patients had daily CT scans beyond the first 2 days of admission.

\section{Radiographic Clearance of Blood from the Ventricular System}

Clearance of blood from the 3 rd and 4 th ventricles, as visualized on the CT scan, occurred at a median of 4 days in the UK group compared to 11 days in the Pcb group $(p=0.02)$. Several patients in both treatment groups had episodes of elevated ICP ( $>20 \mathrm{~mm} \mathrm{Hg}$ ) after the ventricular system was cleared of blood and after having stable low ICP (table 4). This finding may be due to closing the EVD during attempts to determine whether these patients would tolerate removal of the EVD. However, after radiographic clearance of blood from the ventricles, mean daily ICP remained $<20 \mathrm{~mm} \mathrm{Hg}$ in all patients. In $3 / 5$ Pcb-treated patients, blood was not cleared from the 3rd and 4 th ventricles during the ICP monitoring period. One $\mathrm{Pcb}$ patient required a ventriculoperitoneal shunt for communicating hydrocephalus.

\section{Cerebral Perfusion Pressure}

$\mathrm{CPP}$ was rarely below $70 \mathrm{~mm} \mathrm{Hg}$. Baseline CPP ranged from 65 to $133 \mathrm{~mm} \mathrm{Hg}$ (mean $98 \pm 6$ ). Mean CPP for the first 5 days of ICP monitoring was $103 \pm 16 \mathrm{~mm} \mathrm{Hg}$ in the UK group and $103 \pm 18$ in the Pcb group. In the univariate analysis CPP levels decreased significantly with time (over the first 5 days) in the UK group only ( $p=$ $0.05)$. However, CPP levels were not significantly different between groups $(\mathrm{p}=0.88)$. CPP was generally high without specific intervention. Low CPP events $<60$ and $<70 \mathrm{~mm} \mathrm{Hg}$ occurred on only 1 and 6 occasions, respectively. Four of these were associated with elevated ICP (>20 mm Hg), 3 with low mean arterial pressure.

\section{Outcome}

Admission GCS was the same in both groups $(8 \pm 2)$. Median 30-day GOS score was 3 in both groups. One Pcb and 2 UK patients had favorable outcomes (GOS 1 or 2). One patient in the Pcb group died on day 3 after 4 injections of study agent after withdrawal of life-sustaining therapy. There were no other deaths in the first 30 days. The number of high ICP events was not associated with 30-day GOS in this cohort (Spearman's rho: 0.36; $\mathrm{p}=0.28$ ).

\section{Discussion}

In this exploratory analysis of a trial dataset, we demonstrate that significant ICP elevation $>20 \mathrm{~mm} \mathrm{Hg}$ occurs in $14 \%$ of readings after initiation of external CSF drainage in a cohort of 11 patients with severe IVH treated by randomization with intraventricular UK or Pcb. With minor exceptions, the insertion of the EVD was equally effective in controlling ICP in these 2 groups. These findings provide evidence of the value of ICP monitoring in severe IVH. In most subjects, we did not detect significant ICP elevation during temporary interruption of CSF drainage for injection of study agent, suggesting that this method of drug injection for treatment of IVH is safe in terms of ICP management. One subject did routinely experience significant ICP elevation with closure.

It is often assumed that coma and death associated with IVH result from an acute rise in ICP that injures the reticular activating system or compromises cerebral perfusion. Nevertheless, no correlation between ICP and outcome has been reported for IVH, even though greater volumes of intraventricular blood and a greater degree of ventricular dilatation on early CT scans are associated with worse outcomes $[8,9,16]$. An exploratory retrospective analysis of ICP elevation in IVH has been made on convenience samples of IVH patients. Adams and Diringer [10] reported on early control of ICP in 22 patients with supratentorial ICH and hydrocephalus treated with EVD. Emergency CSF drainage controlled initial ICP well in 20/22 patients, but ventricular size did not decrease nor did the level of consciousness improve. Continuous ICP recordings were not analyzed. Diringer et al. [16] also reported that in 81 patients with supratentorial $\mathrm{ICH}$, hydrocephalus was independently associated with higher intubation rates and increased mortality, but outcomes were not different in patients treated with ventriculostomy. Coplin et al. [11] reported on a cohort of 40 patients with spontaneous IVH who received EVDs. The mean initial ICP was $15.6 \mathrm{~mm} \mathrm{Hg}$, and only 6 patients (15\%) had ICP elevation (ICP >20 $\mathrm{mm} \mathrm{Hg}$ ) at the time of EVD placement. ICP elevation at presentation was not associated with a poor GOS.

These results all support the concept that acute obstructive hydrocephalus remains a potentially lethal complication of IVH. These and our data should not be interpreted to suggest that EVD placement and ICP management are unnecessary. Rather they demonstrate that having the EVD open at a certain height [5] which allows for continuous drainage of CSF may contribute to normalization of the ICP. None of the data preclude the po- 
tential harmful effect of intracranial hypertension during the period between the onset of IVH and insertion of the EVD. Nor do they preclude the obvious harmful effects of ICP were EVDs not to be employed. The absence of ICP events may reflect the therapy intensity level, as our study was not designed to assess ICP in the absence of CSF drainage. It is possible that CSF drainage alone via the EVD represents adequate and appropriate management of ICP in spontaneous IVH. However, lysis of intraventricular blood by the additional administration of intraventricular UK appears to shorten the duration of EVD management, likely by more rapid restoration of normal CSF circulation. Intraventricular UK administration did not appear to be associated with improved outcomes in this small cohort.

Although intracranial hypertension was not a common occurrence in our IVH study, the proportion of high ICP events was arithmetically greater in the Pcb-treated patients on all days except day 4 . It is possible that the use of UK prevented some transient ICP fluctuations secondary to more rapid clearance of blood from the ventricular system or by preventing EVD occlusion. Torres et al. [17] reported higher ventriculostomy obstruction rates and a higher incidence of intracranial hypertension in a historic control group compared with 14 patients treated with intraventricular UK for moderate to severe IVH.

In this study the 5th day of ICP monitoring was associated with the highest proportion of ICP events. This occurred after the opening of the $3 \mathrm{rd}$ and 4 th ventricles in all UK-treated patients, but not in Pcb patients. One explanation is that the EVD was closed for the patients with open 3rd and 4th ventricles in anticipation of EVD removal, and that higher ICP was either clinically tolerated by the patient or permitted by the treating physician. It is also possible that with the EVD closed, ICP was monitored continuously rather than intermittently, resulting in detection of more ICP events. This analysis assumed that ICP was low and stable once the EVD was removed. Although no EVD was removed until the patient had low stable ICP for $24 \mathrm{~h}$ with the EVD closed, this assumption may not hold true for all patients.

This is a small sample of patients and cannot capture all the complex or interactive effects of ICP and other variables on outcome. Data collection was not continuous, so transient ICP elevations may not have been detected by the 4-hour or 6-hour reporting method used. The assumption that ICP readings at discrete time points reflect ICP for the entire interval between readings may be adequate for patients with low stable ICP readings, but may not apply to patients with elevated ICP. All analyses are also biased by the need for therapeutic intervention. The protocolized approach required aggressive treatment for any elevated ICP which may also contribute to low numbers of ICP events in this study. The results cannot be generalized to patients with larger ICH volumes who may have impaired compliance or more significant intracranial hypertension. The lack of adjustment for multiple comparisons in this exploratory analysis implies an increased chance of finding an apparent relationship in the sample purely by chance when no relationship exists in the population. Large datasets and prospective data will be required to fully demonstrate any possible relationship.

This prospective analysis of ICP during EVD of CSF in IVH (with or without intraventricular UK) suggests that ICP may not be the ultimate determinant of neurological injury in spontaneous IVH. EVD placement is, however, a life-saving procedure. The finding of normal ICP readings may not necessarily translate into absence of neurological injury. ICP should be monitored in all IVH patients during drainage and during administration of intraventricular drugs so that elevated ICP can be treated rapidly and EVDs can be managed optimally.

The apparent beneficial effect of intraventricular UK on ICP, duration of EVD use, and tolerance to EVD closure implies a therapeutic advantage of this class of therapy in the management of severe IVH.

\section{Acknowledgments}

This work was supported in part by the Eleanor Naylor Dana Fellowship (DFH and WCZ), American Academy of Neurology (WCZ), NIH/NINDS 1RO1 NS 24282-08 (DFH), Grant FD-R001693-01-1 from the Food and Drug Administration Orphan Products Development Agency (DFH), and the France Merrick Foundation Grant (DFH).

References

Cerebrovasc Dis 2009;27:403-410 409 de Weerd AW: The prognosis of intraventricular hemorrhage. J Neurol 1979;222:46-51.

$\checkmark 2$ Graeb DA, Robertson WD, Lapointe JS, Nugent RA, Harrison PB: Computed tomographic diagnosis of intraventricular hemorrhage. Etiology and prognosis. Radiology 1982;143:91-96.

- 3 Todo T, Usui M, Takakura K: Treatment of severe intraventricular hemorrhage by intraventricular infusion of urokinase. J Neurosurg 1991;74:81-86.

Intracranial Pressure and 
4 Bhattathiri PS, Gregson B, Prasad KS, Mendelow AD, STICH Investigators: Intraventricular hemorrhage and hydrocephalus after spontaneous intracerebral hemorrhage: results from the STICH trial. Acta Neurochir 2006;96(suppl):65-68.

5 Steiner T, Diringer MN, Schneider D, Mayer SA, Begtrup K, Broderick J, Skolnick BE, Davis SM: Dynamics of intraventricular hemorrhage in patients with spontaneous intracerebral hemorrhage: risk factors, clinical impact, and effect of hemostatic therapy with recombinant activated factor VII. Neurosurgery 2006;59:767-773.

6 Naff NJ, Carhuapoma JR, Williams MA, Bhardwaj A, Ulatowski JA, Bederson J, Bullock R, Schmutzhard E, Pfausler B, Keyl PM, Tuhrim S, Hanley DF: Treatment of intraventricular hemorrhage with urokinase: effects on 30-day survival. Stroke 2000;31: 841-847.

7 Steinke W, Sacco RL, Mohr JP, Foulkes MA, Tatemichi TK, Wolf PA, Price TR, Hier DB: Thalamic stroke. Presentation and prognosis of infarcts and hemorrhages. Arch Neurol 1992;49:703-710.
8 Mayfrank L, Lippitz B, Groth M, Bertalanffy H, Gilsbach JM: Effect of recombinant tissue plasminogen activator on clot lysis and ventricular dilatation in the treatment of severe intraventricular haemorrhage. Acta Neurochir 1993;122:32-38.

-9 Tuhrim S, Horowitz DR, Sacher M, Godbold $\mathrm{JH}$ : Volume of ventricular blood is an important determinant of outcome in supratentorial intracerebral hemorrhage. Crit Care Med 1999;27:617-621.

10 Adams RE, Diringer MN: Response to external ventricular drainage in spontaneous intracerebral hemorrhage with hydrocephalus. Neurology 1998;50:519-523.

11 Coplin WM, Vinas FC, Agris JM, Buciuc R, Michael DB, Diaz FG, Muizelaar JP: A cohort study of the safety and feasibility of intraventricular urokinase for nonaneurysmal spontaneous intraventricular hemorrhage. Stroke 1998;29:1573-1579.

12 Naff NJ, Hanley DF, Keyl PM, Tuhrim S, Kraut M, Bederson J, Bullock R, Mayer SA, Schmutzhard E: Intraventricular thrombolysis speeds blood clot resolution: results of a pilot, prospective, randomized, doubleblind, controlled trial. Neurosurgery 2004; 54:577-583.
13 The Brain Trauma Foundation. The American Association of Neurological Surgeons. The Joint Section on Neurotrauma and Critical Care. Intracranial pressure treatment threshold. J Neurotrauma 2000;17:493-495.

14 The Brain Trauma Foundation. The American Association of Neurological Surgeons. The Joint Section on Neurotrauma and Critical Care. Guidelines for cerebral perfusion pressure. J Neurotrauma 2000;17:507-511.

15 Steiner L, Bergvall U, Zwetnow N: Quantitative estimation of intracerebral and intraventricular hematoma by computer tomography. Acta Radiol 1975;346(suppl):143154.

16 Diringer MN, Edwards DF, Zazulia AR: Hydrocephalus: a previously unrecognized predictor of poor outcome from supratentorial intracerebral hemorrhage. Stroke 1998;29: 1352-1357.

-17 Torres A, Plans G, Martino J, Godino O, Garcia I, Gracia B, Acebes JJ: Fibrinolytic therapy in spontaneous intraventricular haemorrhage: efficacy and safety of the treatment. Br J Neurosurg 2008;22:269-274. 